\title{
Detection of antibodies to denatured Human Leucocyte Antigen molecules by
}

\section{Single Antigen Luminex}

\section{Short title: Antibodies to denatured HLA by Luminex}

María Gutiérrez-Larrañaga ${ }^{1,2}$, MD; Laura Riesco ${ }^{1,2}$, MD; Sandra Guiral1, 2, MD; Juan Irure ${ }^{1,2}$, MD; Emilio Rodrigo ${ }^{3}, \mathrm{PhD}$; J.Gonzalo Ocejo-Vinyals ${ }^{1,2}$, PhD; Jaume

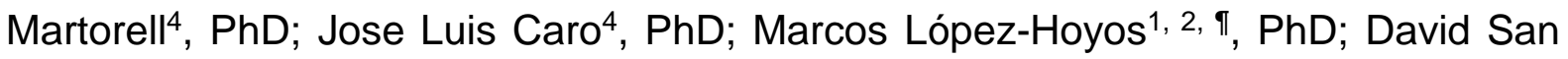
Segundo ${ }^{1,2, \uparrow, *}$, PhD.

${ }^{1}$ Immunology Department. University Hospital Marqués de Valdecilla-IDIVAL, Santander, Spain.

${ }^{2}$ Tissue Typing Laboratory. University Hospital Marqués de Valdecilla, Santander, Spain.

${ }^{3}$ Nephrology Department. University Hospital Marqués de Valdecilla-IDIVAL, Santander, Spain.

4Immunology Department, Hospital Clínic, Barcelona, Spain;

IThese authors contributed equally to this work

* Correspondence: David San Segundo; Mailing address: Immunology department of University Hospital Marques de Valdecilla, Valdecilla Avenue s/n, Santander, Spain. E-mail: dsansegundo@humv.es; Tel.: +34 942202549 (DSS). 
Pages: 20

Tables:2

Figures:2

Appendices: 3 tables and 2 figures

This article is protected by copyright. All rights reserved. 


\section{Abbreviations}

Ab, antibody

ABMR, antibody mediated rejection

AM, manufacturer $A$

AT1R, angiotensin II type 1 receptor.

B2M, beta 2-microglobulin.

$\mathrm{BM}$, manufacturer $\mathrm{B}$.

CDC, complement-dependent cytotoxicity.

dHLA, denatured-HLA.

DSA, donor-specific antibodies.

EDTA, ethylenediaminetetraacetic acid.

FCXM, flow cytometry cross-match.

HLA, human leukocyte antigen.

MFI, mean fluorescence intensity.

MICA, MHC class I chain-related sequence $A$

nHLA, native-HLA.

PBMCs, peripheral blood mononuclear cells

SA, single antigen.

$\mathrm{SAB}$, single antigen bead.

SSP, sequence specific primer. 


\section{Abstract:}

Introduction: The anti-HLA antibody detection has been improved in sensitivity and specificity with solid-phase antigen bead (SAB) assays based on Luminex. However, false positive results due to denatured HLA may arise after single antigen test. The aim of this study was to compare the performance of the two Luminex technologybased anti-HLA detection kits available in the market in revealing undesired anti-HLA antibody results.

Methods and materials: A prospective cohort was assessed for anti-HLA antibodies with single antigen A manufacturer (AM) kit and a comparison cohort with single antigen $B$ manufacturer (BM) kit.

Results: A total of 11 out of 90 patients in a prospective cohort presented monospecific HLA-I antibodies with AM, and 5 out of 11 confirmed monospecific reaction with BM. Despite the confirmation of monospecific reaction with both manufacturers, $80 \%$ were assigned as denatured HLA reaction by specific crossmatch. Further comparative cohorts detected 4 out of 6 monospecific reactions with BM that were confirmed as possible denatured HLA reactions.

Conclusions: A positive SAB test should rule out a reaction against a denatured HLA molecule, thus avoidance of prolonged waitlist periods or misattribution of antiHLA reactions after transplantation. 
Keywords: HLA antigens / immunology; Antibodies / analysis; Flow cytometry / methods; Microspheres; Cytotoxicity Tests, Immunologic / methods; Histocompatibility testing / methods.

\section{Introduction}

Anti-HLA antibody (Ab) testing experienced a major breakthrough with the development of solid-phase assays based on Luminex technology, increasing the sensitivity and enabling the detection of low levels of HLA-specific Abs ${ }^{1}$. Despite this, several potential pitfalls should be taken into account regarding Luminex anti-HLA Ab detection ${ }^{2}$. Three different kinds of errors could appear. First, there are false positive results due to Ab binding to misfolded HLA proteins. Thus, denaturing of HLA antigens during the processes of purification and bead coating could lead to the generation of neo-epitopes that under physiological circumstances would be inaccessible for Abs. Second, lack of cut-off consensus between laboratories and wide intra- and interassay variability could also result in false positive results. Finally, the prozone effect is a widely accepted source of false negative results ${ }^{3-7}$.

Two types of panels depending on the composition of their target antigen are available. The screening panel has two different bead groups, coated with either HLA class I or class II protein molecules obtained from multiple individual cell lines, and are used as a screening test for the presence or absence of anti HLA class I/II Abs. However, they 
do not provide accurate information about Ab specificity. The single-antigen beads (SAB) panel has multiple beads, each one coated with a unique protein molecule representing a single cloned HLA antigen which enables the identification of any given antibody specificity, and provides the highest degree of anti-HLA Ab resolution. However, denatured beta 2-microglobulin (B2M)-free heavy chains and structurally different HLA-class-I molecules are present in a single bead which could lead to false positive results ${ }^{8}$, whereas denatured HLA molecule cannot be ruled out in screening panels ${ }^{9,10}$.

The denatured HLA (dHLA) reaction has been detected not only in sensitized transplant patients but also in unsensitized males ${ }^{10}$. The presence of denatured HLA reactions was detected in more than $30 \%$ of sensitized patients ${ }^{11}$. However there are few reports assessing the clinical impact of the presence of dHLA in the transplant setting $12,13,14$.

The main objective in anti-HLA Ab testing should be the detection of Abs against a native HLA molecule. Two manufacturers provide anti-HLA Ab tests based on Luminex technology. Several works have raised differences between them. Some authors have identified a greater proportion of denatured HLA-I antigens on the surface of beadsets in One lambda SAB kits, whereas Immucor SAB kits present most of its antigens in the native trimeric form composed of HLA-I heavy chain, B2M and peptide, as evidenced by monoclonal Ab binding ${ }^{15}$. The main goal of this study was to analyze 
and compare the performance of the two Luminex technology-based anti-HLA detection kits on revealing unexpected anti-HLA antibody results.

\section{Materials and Methods}

\subsection{Patient selection}

The anti-HLA antibody test is performed within routine sampling in patients while listed and after solid organ transplant monitoring. The patients gave their written consent once included on the waiting list. The study was approved by the Local Ethics Committees and conducted in accordance with the Declaration of Helsinki.

\subsubsection{Prospective cohort}

A total of 90 serum samples with negative results after anti-HLA Ab screening assay (manufacturer A) were recruited for the study during one year in University Hospital Marqués de Valdecilla (Santander, Spain). Forty out of 90 (44.4\%) sera came from solid organ transplant candidates newly included in the institution's waiting list whereas $50(55.5 \%)$ sera came from solid organ transplant patients followed posttransplant within one year. A subsequent assessment of samples rendered negative after screening but positive on SAB assay results were selected for further analysis (i.e., acid treatment of the beads, CDC and FCXM as described below). Finally, 11 sera with confirmed negative screening results but positive monospecific anti-HLA class-I results were selected. Their main demographic and immunological parameters 
are summarized in Appendix Table 1. The 11 samples were chosen in order to facilitate the selection of donor HLA-typed cells for testing each monospecific HLA reactive sera.

In order to elucidate if such findings may be differentially affecting the performance of the kits on detecting unexplained anti-HLA results on SAB assays, we reanalyzed them with B manufacturer's anti-HLA Ab kit.

\subsubsection{Comparison cohort}

Serum samples from 6 patients with confirmed negative anti-HLA screening and positive anti-HLA class I monospecific reaction in SAB assay (manufacturer B) not explained by previous sensitization events were selected in the Clinic Hospital (Barcelona, Spain) and reanalyzed using AM kit. The main demographic and immunological parameters of the patients for potential allosensitization are summarized in Appendix Table 2.

An overview of the study is detailed in Appendix Figure 1.

\subsection{Anti-HLA antibody test}

The presence of anti-HLA Abs was tested by using screening and SAB assays from the only two manufacturers available as described in the text (LABScreen Mixed, Cat LSM12, Lot: 020; LABScreen Single Antigen Class I, Cat LS1A04, Lot: 010; and LABScreen Single Antigen Class II, Cat LS2A01, Lot 012, One Lambda, Canoga Park, CA, United States) referred as A manufacturer; and (Lifescreen Deluxe, Cat LMX, Lot 
3006878; LSA class I, Lot 3006346 and LSA class II, Lot 3006494, Immucor, Gateway Drive, GA, United States) referred as B manufacturer and following manufacturers' instructions; and analyzed on a Luminex platform (LabScan100). The cut-off value was set at 500 baseline MFI for screening and 1,500 baseline MFI for SAB test . Such a difference in the cut-off is intended to minimize false negative results in anti-HLA Ab screening and false positive results in SAB assays. We considered sera with values between 1,500 and 3,000 baseline MFI as weakly positive.

\subsection{In vitro anti-HLA reactivity against denatured vs. native HLA-molecule}

In order to decipher the reaction against denatured (dHLA) or native HLA-molecule ( $\mathrm{nHLA}$ ), in samples analyzed with AM kit rendering negative screening but positive $\mathrm{SAB}$ results, acid treatment of the $\mathrm{SAB}$ beadsets was performed as previously described $^{11}$. Briefly, the beads were treated with $1 \mathrm{~mL}$ of $1 \%$ bovine serum albumin and $0.1 \mathrm{M}$ glycine buffer $(\mathrm{pH}=2.8)$ during 30 minutes, then washed twice with the wash buffer provided by manufacturer prior to sera incubation and subsequently followed the manufacturer's instructions. The denatured beads were confirmed with anti-w6/32phycoerythrin monoclonal antibody (Biolegend, San Diego, CA), a representative example of acid treatment of the beads is shown (Appendix Figure 2). Because there is no consensus for a cut off value of MFI after acid treatment, we decided to set at 1,500 MFI. Values between 1,500 and 3,000 MFI were considered as weakly positive. The MFI values pre and post acid treatment are described in Appendix Table 3.

\subsection{Ex vivo anti-HLA reactivity}


Cytotoxic (CDC) and flow cytometry cross match (FCXM) were performed when possible in samples with negative results for screening while positive SAB results using A manufacturer kit against reactive HLA typed cells. CDC was conducted as previously described ${ }^{16}$. For FCXM, peripheral blood mononuclear cells (PBMCs) isolated by Ficoll gradient were incubated with rat serum (Sigma Aldrich, St. Louis, MO) during 20 minutes to avoid unspecific binding (Cytometry A. 2016;89(11):10011009). Undiluted and $1 / 10$ diluted serum was added to PBMCs and incubated during 30 minutes at room temperature. Anti-CD3 ECD (clone UCHT-1, Beckman Coulter, Brea, CA), CD19 PC5 (clone J3-119 Beckman Coulter, Brea, CA) and subsequently Fab'-IgG FITC (Immunostep, Salamanca, SPAIN) were added and samples were acquired using a Navios EX Flow Cytometer (Beckman Coulter, Brea, CA). Flow cytometry crossmatch for class-I antigens was considered positive in the CD3+ gate when the ratio of median fluorescence value serum / median fluorescence value negative control was > 1.5; for class-II antigens, the FCXM was considered positive in $\mathrm{CD} 19+$ gate when the ratio was $>2.0$.

The HLA typing of the donors was performed for HLA loci A, B, DRB1, DQA1 and DQB1 by high resolution SSP (Life Technologies, Brown Deer, WI) to confirm reaction against nHLA.

\subsection{Statistical analysis}

Kolmogorov-Smirnoff test was used to reveal if the data of baseline MFI followed a normal distribution. The Mann Whitney test was used to compare median baseline 
MFI. All tests were performed using GraphPad software version 7.0 (San Diego, CA). The R software and "ggplot2" package have been used for graphics.

\section{Results}

\subsection{Single Antigen positive reactions against denatured or native HLA molecules}

A total of 21 patients from a prospective cohort (23.3\%) showed positive SAB results (both in HLA class-I and class-II specificities) with negative screening test result. For further analysis we focused only on the 11 patients (9.9\%) with positive reactions only against HLA class-I for simplicity. Five out of 11 (45.4\%) were transplanted patients, although none of the monospecific reactions were donor-specific. Of note, only two samples were from unsensitized males. A summary of potential sensitization of the patients is summarized in appendix Table 1. Upon acid treatment the HLA antigens attached to the beads were denatured; a negative result after SAB of acid-treated beads would suggest a native-HLA (nHLA) reaction. However, a total of 11/11 (100\%) patients had positive reactions after bead-treatment (Figure 1), so denatured-HLA (dHLA) reaction could not be ruled out. Moreover, to confirm the reactivity of the anti-HLA Abs, both CDC and FCXM against a limited number of specific HLA-typed cells were performed. As expected, all the samples with potential anti-dHLA reactions that could be tested in crossmatch rendered both CDC and FCXM negative results, summarized in Tables 3 and 4. 


\subsection{Comparison of baseline MFI from screening vs single antigen test}

The SAB test was performed with sera from a prospective cohort with a previous negative result on screening test. Once a positive result in HLA class-I was found, we calculated the mean baseline MFI of the bead groups where the positive HLA antigen was included (i.e. $A^{\star 11: 01}$ is present in beads \#008, \#014, \#015 and \#016 in the screening full panel of Lot 020 from One Lambda and in bead\#CLI02, \#CLI04, \#CLI05, \#CLI06 panel of Lot 3006878 from Immucor). The Figure 2 describes the MFI values obtained after screening and Single Antigen test of the sera.

\subsection{Confirmation of non-concordant results with B manufacturer's kit}

In order to clarify the monospecific reactions observed with A manufacturer (AM), 11 serum samples of patients with confirmed negative screening results and positive antiHLA class I monospecific reactions with AM kit were selected for further analysis using B manufacturer's (BM) kit. Seven out of the 11 serum samples (58.3\%) continued displaying negative screening but positive anti-HLA Class I SAB assay results, although a significant reduction of median (interquartile range) baseline MFI levels (AM vs BM) $3,877(3,633-6,101)$ vs $1,405(100-2,555)$, was observed $(p<0.0001)$. In this comparative study the anti-HLA reactions of samples rendering negative crossmatch results were considered to be directed against denatured HLA antigens on the beads. The comparative results between the two manufacturers are summarized in Table 1. 


\subsection{Monospecific reactions observed with B manufacturer- comparison cohort}

Six serum samples from the comparison cohort were found to repeatedly show negative screening but positive monospecific anti-HLA Class I SAB assay results when analysed employing BM kit. One out of six (16.7\%) confirmed such results by analysis using AM kit, while the other five rendered negative results in both screening and SAB assays with AM kit. The comparative results between the two manufacturers are summarized in Table 2.

\section{Discussion}

The anti-HLA Ab test is mandatory prior to any solid organ transplantation in order to quantify the level of allosensitization. The SAB-assay itself has several pitfalls and a proper monitoring of anti-HLA Abs should be performed while the patient is included on the waiting list. The guidelines indicates monitoring every 3 months in all solid organ waiting list patients ${ }^{17}$. Patients that have lost a previous transplant, women potentially sensitized due to pregnancy or abortions and transfused patients are potential candidates to identify reactions against HLA antigens. Our idea was to better identify this kind of patients by performing directly SAB assay as first option instead of antiHLA Abs screening. However, an increased number of missassigned-HLA specificities could lead to unacceptable mismatches with potential donors and prolonged periods on waiting list. Moreover, testing anti-HLA Abs by SAB assays in transplant monitoring could assign a patient with donor-specific anti-HLA antibodies (DSA), when the 
identified antibodies could actually be directed against dHLA, with uncertain impact on the allograft. The interference with complement and prozone effect could be avoided with serum EDTA-treatment.

Deciphering the reactivity of the anti-HLA Abs between denatured or native HLA would help in the identification of deleterious anti-HLA Abs, with different implications if on waiting list or post-transplant monitoring. In the first case, it would potentially increase the time on waiting list, whereas in transplanted patients, humoral rejection diagnosis requires a confirmatory biopsy with circulating DSA. Thus, the identification of the found antibodies as directed against denatured HLA would avoid treatment of nonrelevant DSA. However, with clinical and/or histological findings, other means of humoral rejection mediated by non-HLA Abs (MICA, AT1R, endothelial cells, and others) should be ruled out ${ }^{18-20}$.

The presence of anti-HLA Abs in patients not exposed to any allosensitizing event due to cross-reaction with viral epitopes has been documented ${ }^{21,}{ }^{22}$. However, the literature is mixed with recent reports identifying no cross reactivity with viral and allo antibodies ${ }^{23}$.

For this reason, a proper assessment of the nature of these antibodies is of great importance for their clinical implication. Pre-transplant anti-dHLA DSA reactions have been associated with negative FCXM, low risk of acute antibody-mediated rejection (ABMR) ${ }^{13}$ and better long-term graft survival ${ }^{12}$. It should be taken into account the different anti-HLA detection level of FCXM and SAB assay. In our study, no clinical 
assessment was performed but recently kidney transplant recipients with anti-dHLACw DSA showed negative FCXM reactions, lower ABMR rate and better graft survival 14. The HLA-Cw is expressed at a lower frequency on cells used within FCXM, but is at a high density on the SAB assay. This could lead discrepant results. In our study, most of the monospecific reactions selected had more than $3000 \mathrm{MFI}$ with a negative FCXM which supports the reaction against denatured HLA. However, a limitation of the work is that, in order to demonstrate reaction against dHLA, only acid treatment in prospective cohort was performed. Previously, several groups also confirmed the assessment with iBeads with good correlation with acid treatment ${ }^{24}$.

In patients waiting for heart or lung transplantation, the definition of forbidden HLA antigens should be accurate due to virtual crossmatch and lack of prospective crossmatch performance. Cao S. et al have performed direct SAB in lung-waiting list patients and identified more anti-HLA antibodies compared with screening assays in samples rendering negative crossmatches ${ }^{25}$. These observations are consistent with our findings; however the screening and SAB assays have different source of HLA molecules coating the beads, the screening panel is manufactured from nonrecombinant cell line whereas SAB panel with recombinant cell lines denaturing differently and present on the surface of the beads at different densities providing potential discrepant results.

Since there are two different manufacturers of SAB assay for anti-HLA antibody testing, several studies have compared the results provided by both of them, with no 
substantial differences ${ }^{26}$. Some discordant results could be explained by different HLA-load constructs on the beads ${ }^{15}$. A recent study compared the performance and accuracy of the only two commercially available Luminex-based SAB assays in the ABMR setting. Authors concluded that both kits, despite significant differences in their sensitivity and specificity, had a good correlation ${ }^{27}$.

Our results show that most of confirmed mono-specific HLA class-I reactions from unsensitized patients by $A M$ and BM render both CDC and FCXM negative results that point to denatured-HLA reactions and could be due to similar way of HLA antigenload on beads in their manufacture.

In the present study, all mono-specific reactions detected by SAB, irrespectively of baseline MFI levels, render negative CDC and FCXM suggesting dHLA reactions. Although it should be taken into account that the different sensitivity of the techniques could justify some discordant results between SAB test and crossmatch ${ }^{28}$.

Upon these results, we propose that a positive monospecific reaction detected by SAB in a patient without history of sensitizing events should be defined as native HLA, after a negative reaction with acid treatment of the beads, CDC and/or FCXM against donor cells with the type of interest, and, besides the sample should be studied with the kit of the other manufacturer, which could have logistic problems in laboratory organization.

The main limitation of this study is the scarce number of clinical cases to discuss after transplantation; though the study was driven, in part, from a technical laboratory point 
of view. The results were performed in a single mixed beads lot, however, the confirmation in different bead lots would improve the robustness of our results. Another limitation was the lack of CDC and FCXM for all anti-HLA reactivities detected, most of them due to their low frequency in our population $(A * 34: 0-1.5 \%, B * 73: 0-2 \%$ and $A \star 80: 0-2 \%$ of European Caucasians) ${ }^{29}$. Moreover, the identification of Abs against low frequency HLA could suggest false positive reactions.

To better rely on anti-HLA Abs tests the efforts tests, efforts should identify potential biases in anti-HLA Ab results, leading to the fine tuning of anti-HLA Ab profiles of the patients which could be of special importance in those who are highly sensitized.

Acknowledgments: We would like to acknowledge Immucor and One Lambda for kindly providing the reagents for anti-HLA Abs testing.

Funding: This research was partially supported by grant from ISCIII - REDinREN RD16/0009/0027. 


\section{References}

1. Tait BD, Hudson F, Cantwell L, et al. Review article: Luminex technology for HLA antibody detection in organ transplantation. Nephrology. Apr 2009;14(2):247-254.

2. Tait BD, Hudson F, Brewin G, Cantwell L, Holdsworth R. Solid phase HLA antibody detection technology--challenges in interpretation. Tissue antigens. Aug 2010;76(2):87-95.

3. Tambur AR, Herrera ND, Haarberg KM, et al. Assessing Antibody Strength: Comparison of MFI, C1q, and Titer Information. American journal of transplantation : official journal of the American Society of Transplantation and the American Society of Transplant Surgeons. Sep 2015;15(9):2421-2430.

4. Schnaidt M, Weinstock C, Jurisic M, Schmid-Horch B, Ender A, Wernet D. HLA antibody specification using single-antigen beads--a technical solution for the prozone effect. Transplantation. Sep 15 2011;92(5):510-515.

5. Schwaiger E, Wahrmann M, Bond G, Eskandary F, Bohmig GA. Complement component C3 activation: the leading cause of the prozone phenomenon affecting HLA antibody detection on single-antigen beads. Transplantation. Jun 27 2014;97(12):1279-1285.

6. Visentin J, Vigata M, Daburon S, et al. Deciphering complement interference in anti-human leukocyte antigen antibody detection with flow beads assays. Transplantation. Sep 27 2014;98(6):625-631.

7. Zachary AA, Lucas DP, Detrick B, Leffell MS. Naturally occurring interference in Luminex assays for HLA-specific antibodies: characteristics and resolution. Human immunology. Jul 2009;70(7):496-501.

8. Michel K, Santella R, Steers J, et al. Many de novo donor-specific antibodies recognize beta2 microglobulin-free, but not intact HLA heterodimers. Hla. May 2016;87(5):356-366.

9. Sullivan HC, Gebel HM, Bray RA. Understanding solid-phase HLA antibody assays and the value of MFI. Human immunology. Jul - Aug 2017;78(7-8):471-480.

10. El-Awar N, Terasaki PI, Nguyen A, et al. Epitopes of human leukocyte antigen class I antibodies found in sera of normal healthy males and cord blood. Human immunology. Oct 2009;70(10):844-853.

11. Visentin J, Guidicelli G, Bachelet T, et al. Denatured class I human leukocyte antigen antibodies in sensitized kidney recipients: prevalence, relevance, and impact on organ allocation. Transplantation. Oct 15 2014;98(7):738-744.

12. Visentin J, Marroc M, Guidicelli G, et al. Clinical impact of preformed donor-specific denatured class I HLA antibodies after kidney transplantation. Clinical transplantation. May 2015;29(5):393-402.

13. Otten HG, Verhaar MC, Borst HP, et al. The significance of pretransplant donor-specific antibodies reactive with intact or denatured human leucocyte antigen in kidney transplantation. Clinical and experimental immunology. Sep 2013;173(3):536-543.

14. Visentin J, Bachelet T, Aubert O, et al. Reassessment of the clinical impact of preformed donor-specific anti-HLA-Cw antibodies in kidney transplantation. American journal of transplantation : official journal of the American Society of Transplantation and the American Society of Transplant Surgeons. May 2020;20(5):13651374.

15. Ravindranath $\mathrm{MH}$, Jucaud V, Ferrone S. Monitoring native HLA-I trimer specific antibodies in Luminex multiplex single antigen bead assay: Evaluation of beadsets from different manufacturers. Journal of immunological methods. Nov 2017;450:73-80.

16. Irure J, Asensio E, Rodrigo E, et al. Improvement in the definition of anti-HLA antibody profile in highly sensitized patients. PloS one. 2017;12(2):e0171463.

17. G. Karam TK. Guidelines on Renal Transplantation. European Association of Urology 2013.

18. Dragun D, Catar R, Philippe A. Non-HLA antibodies against endothelial targets bridging allo- and autoimmunity. Kidney international. Aug 2016;90(2):280-288. 
19. Reinsmoen NL. Immunological Risk Stratification by Assessing Both the HLA and Non-HLA-Specific Antibodies: Time to Include Testing for Non-HLA Antibodies in the Routine Clinical Antibody Analysis Profile? Transplantation. Jan 2017;101(1):23-25.

20. Reinsmoen NL, Zhang X. Non-human leukocyte antigen-specific antibodies in thoracic transplantation. Current opinion in organ transplantation. Aug 2016;21(4):350-354.

21. D'Orsogna LJ, Roelen DL, Doxiadis, II, Claas FH. Alloreactivity from human viral specific memory Tcells. Transplant immunology. Aug 2010;23(4):149-155.

22. Morales-Buenrostro LE, Terasaki PI, Marino-Vazquez LA, Lee JH, El-Awar N, Alberu J. "Natural" human leukocyte antigen antibodies found in nonalloimmunized healthy males. Transplantation. Oct 27 2008;86(8):1111-1115.

23. Heidt S, Feltkamp MC, Karahan GE, et al. No Evidence for Cross-reactivity of Virus-specific Antibodies With HLA Alloantigens. Transplantation. Nov 2018;102(11):1844-1849.

24. Visentin J, Guidicelli G, Nong T, et al. Evaluation of the iBeads assay as a tool for identifying class I HLA antibodies. Human immunology. Sep 2015;76(9):651-656.

25. Cao S, Courtwright AM, Lamattina AM, et al. The impact of screening method on HLA antibody detection before and after lung transplantation: A prospective pilot study. The Journal of heart and lung transplantation : the official publication of the International Society for Heart Transplantation. Apr 2018;37(4):531-533.

26. Minucci PB, Resse M, Sabia C, Esposito A, De Iorio G, Napoli C. Anti-HLA Antibodies Testing on Solid Phase: Comparative Evaluation of Different Kit Vendors Through Luminex Technology. Experimental and clinical transplantation : official journal of the Middle East Society for Organ Transplantation. Dec 2017;15(6):636640.

27. Bertrand D, Farce F, Laurent C, et al. Comparison of Two Luminex Single-antigen Bead Flow Cytometry Assays for Detection of Donor-specific Antibodies After Renal Transplantation. Transplantation. Mar 2019;103(3):597-603.

28. Visentin J, Bachelet T, Borg C, et al. Reassessment of T Lymphocytes Crossmatches Results Prediction With Luminex Class I Single Antigen Flow Beads Assay. Transplantation. Mar 2017;101(3):624-630.

29. Jucaud V, Ravindranath MH, Terasaki PI. Conformational Variants of the Individual HLA-I Antigens on Luminex Single Antigen Beads Used in Monitoring HLA Antibodies: Problems and Solutions. Transplantation. Apr 2017;101(4):764-777. 
Availability of data: The data that support the findings of this study are available from the corresponding author upon reasonable request. 


\section{Tables:}

Table 1. Comparative results of samples from the prospective cohort.

\begin{tabular}{|c|c|c|c|c|c|c|}
\hline \multicolumn{3}{|c|}{ Class-I } & \multirow[b]{2}{*}{$\mathbf{B}_{M}$} & \multirow[b]{2}{*}{ Reaction } & \multicolumn{2}{|l|}{ Crossmatch } \\
\hline Serum \# & Bead & $A_{M}$ & & & CDC/FCXM & Assignment \\
\hline 1 & B7 & 3,633 & 3,326 & $\mathrm{~T}_{\mathrm{R}}$ & NEGATIVE & Denatured \\
\hline 2 & A23 & 3,877 & 2,555 & $\mathrm{~T}_{\mathrm{R}}$ & NEGATIVE & - \\
\hline 3 & B17 & 6,100 & 1,321 & $\mathrm{~F}_{\mathrm{RA}}$ & NEGATIVE & Denatured \\
\hline 4 & A34 & 3,861 & $<100$ & $\mathrm{~F}_{\mathrm{RA}}$ & NA & - \\
\hline 5 & B73 & 13,574 & 3,199 & $\mathrm{~T}_{\mathrm{R}}$ & NA & - \\
\hline 6 & A11 & 5,645 & $<100$ & $\mathrm{~F}_{\mathrm{RA}}$ & NEGATIVE & Denatured \\
\hline 7 & Cw7 & 3,572 & 1,583 & $T_{R}$ & NEGATIVE & Denatured \\
\hline 8 & A80 & 10,139 & 1,405 & $\mathrm{~F}_{\mathrm{RA}}$ & NA & - \\
\hline 9 & Cw9 & 5,190 & $<100$ & $\mathrm{~F}_{\mathrm{RA}}$ & NA & - \\
\hline 10 & B37 & 2,050 & 1,617 & $\mathrm{~T}_{\mathrm{R}}$ & NA & - \\
\hline 11 & A34 & 3,821 & $<100$ & $\mathrm{~F}_{\mathrm{RA}}$ & NA & - \\
\hline
\end{tabular}


Table 2. Comparative results of samples from comparison cohort.

\begin{tabular}{lllllll}
\hline Serum \# & $\begin{array}{l}\text { Class-I } \\
\text { Bead }\end{array}$ & $\mathbf{A}_{M}$ & $\mathbf{B M}_{M}$ & $\begin{array}{l}\text { Reactio } \\
\mathbf{n}\end{array}$ & $\begin{array}{l}\text { Crossmatch } \\
\text { CDC/FCXM }\end{array}$ & Assignment \\
\hline 12 & A23/A24 & 206 & $\mathbf{3 , 6 9 0}$ & $\mathrm{F}_{\mathrm{RB}}$ & NEGATIVE & Denatured \\
13 & A23/A24 & 66 & $\mathbf{1 , 7 8 7}$ & $\mathrm{F}_{\mathrm{RB}}$ & NEGATIVE & Denatured \\
14 & A23/A24 & 396 & $\mathbf{7 , 4 7 8}$ & $\mathrm{F}_{\mathrm{RB}}$ & NEGATIVE & Denatured \\
15 & A23/A24 & 397 & $\mathbf{3 , 0 9 1}$ & $\mathrm{F}_{\mathrm{RB}}$ & NEGATIVE & Denatured \\
16 & B57/B58 & 92 & $\mathbf{2 , 0 0 5}$ & $\mathrm{F}_{\mathrm{RB}}$ & NA & - \\
17 & B57/B58 & 5,961 & $\mathbf{3 , 0 5 5}$ & $\mathrm{T}_{\mathrm{R}}$ & NA & - \\
\hline
\end{tabular}

The monospecific reaction against A23/A24 and B57/B58 refers to A9 and B17 reactions. The mean fluorescence intensity (MFI)

values are the mean of MFI from the different alleles in each single antigen panel. AM: Manufacturer A; BM: Manufacturer B; NA:

Not available; TR: true reaction (confirmed in both manufacturers); FRB: false reaction B manufacturer. 
Figure legends:

Figure1: Mean Fluorescence Intensity values in single antigen bead and acidtreated bead assays. The mean fluorescence intensity (MFI) values after single antigen bead $(\mathrm{S} A \mathrm{~B})$ assay detecting mono-specific reactions in each patient are shown (A). After acid treatment of the beads as described in Material and Methods section, an increase in MFI values is observed (B). The cut off value in SAB assay was set at $3000 \mathrm{MFI}$ (black line) and in acid-treated bead assay was set at $1500 \mathrm{MFI}$ (dotted line). The arrows in each patient indicate the MFI value of each mono-specific reacion after acid treatment of the beads.

Figure 2: Comparison of median MFI for SAB Class I (white circles) and screening (black squares) test for anti-HLAantibodies in serum with mono-specific reactions. 


\section{Appendix Figure 1. Overview of the study.}

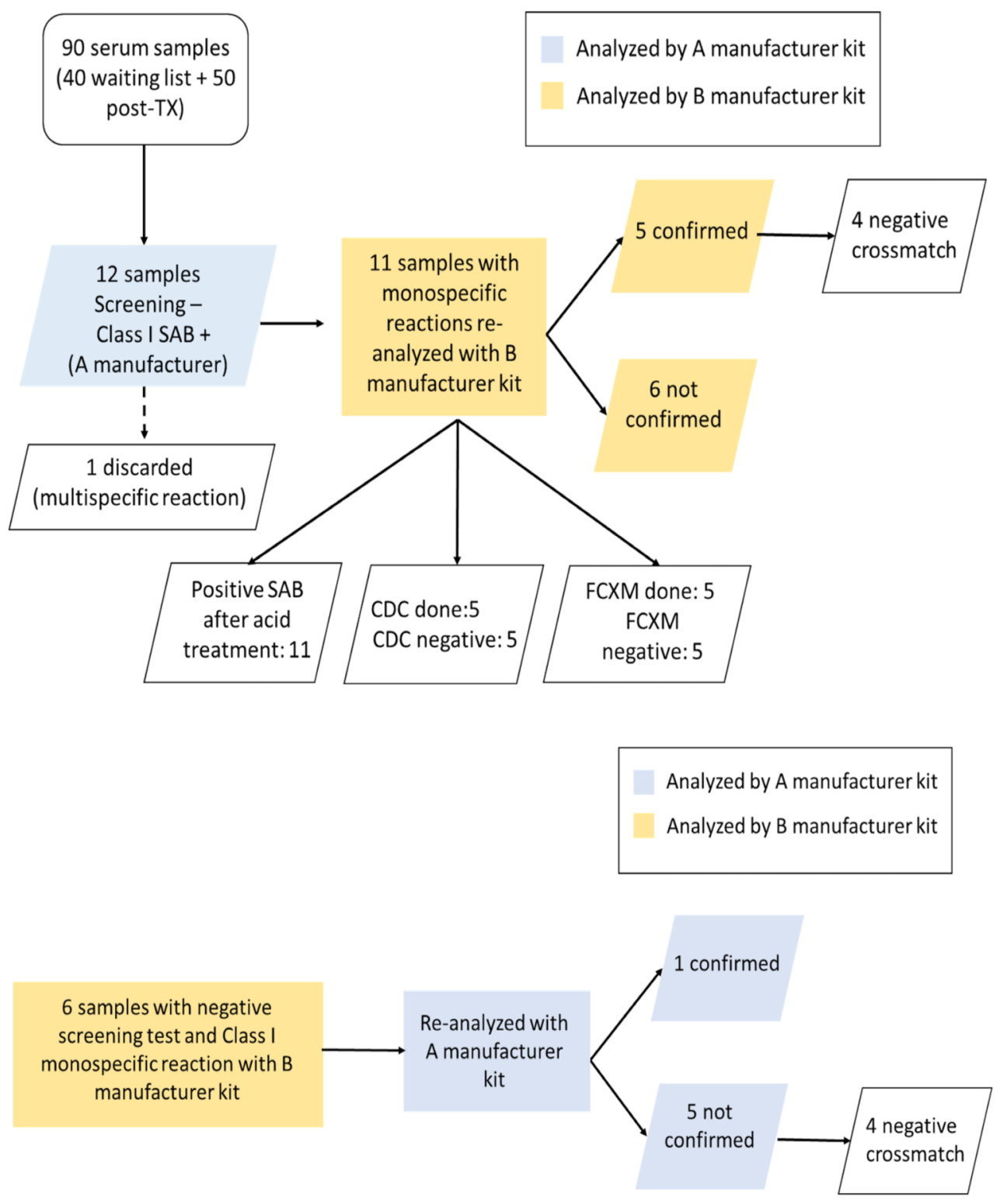




\section{Appendix Figure 2A}

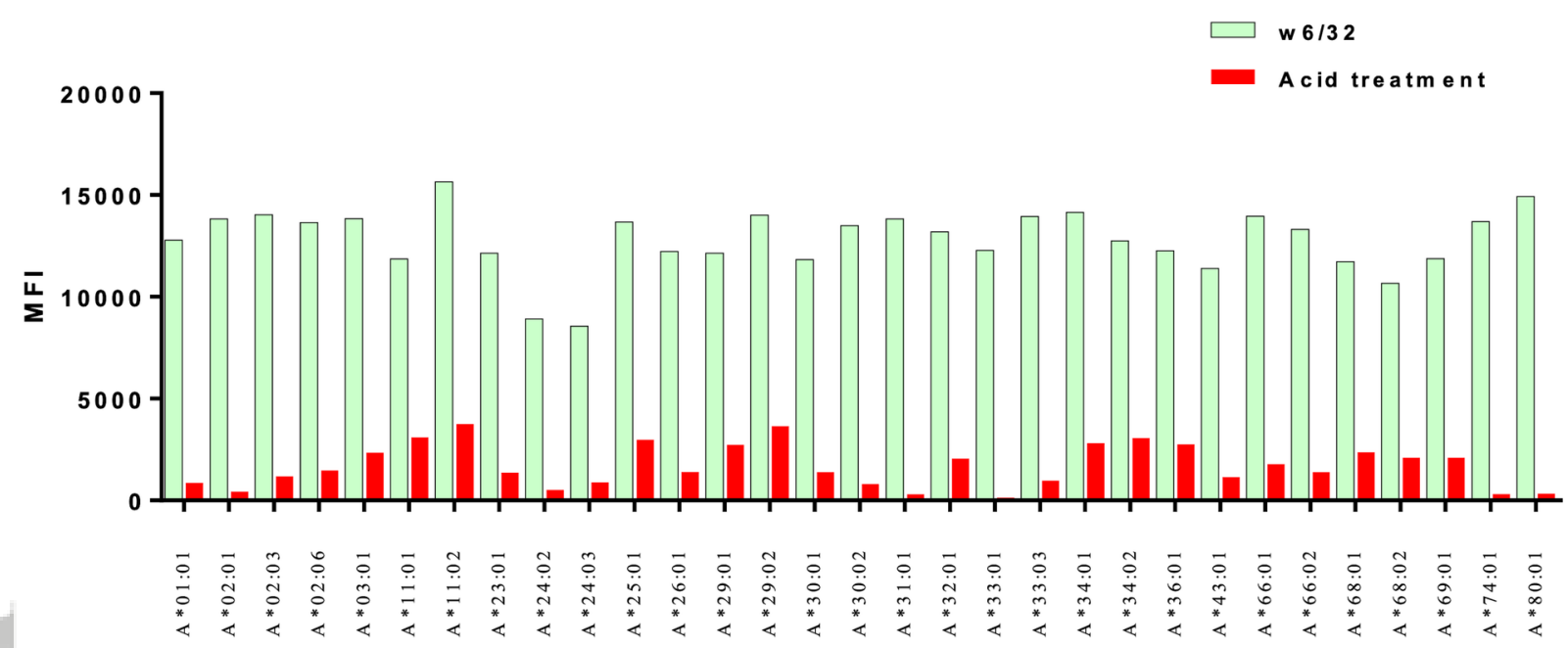

This article is protected by copyright. All rights reserved. 


\section{Appendix Figure 2B}

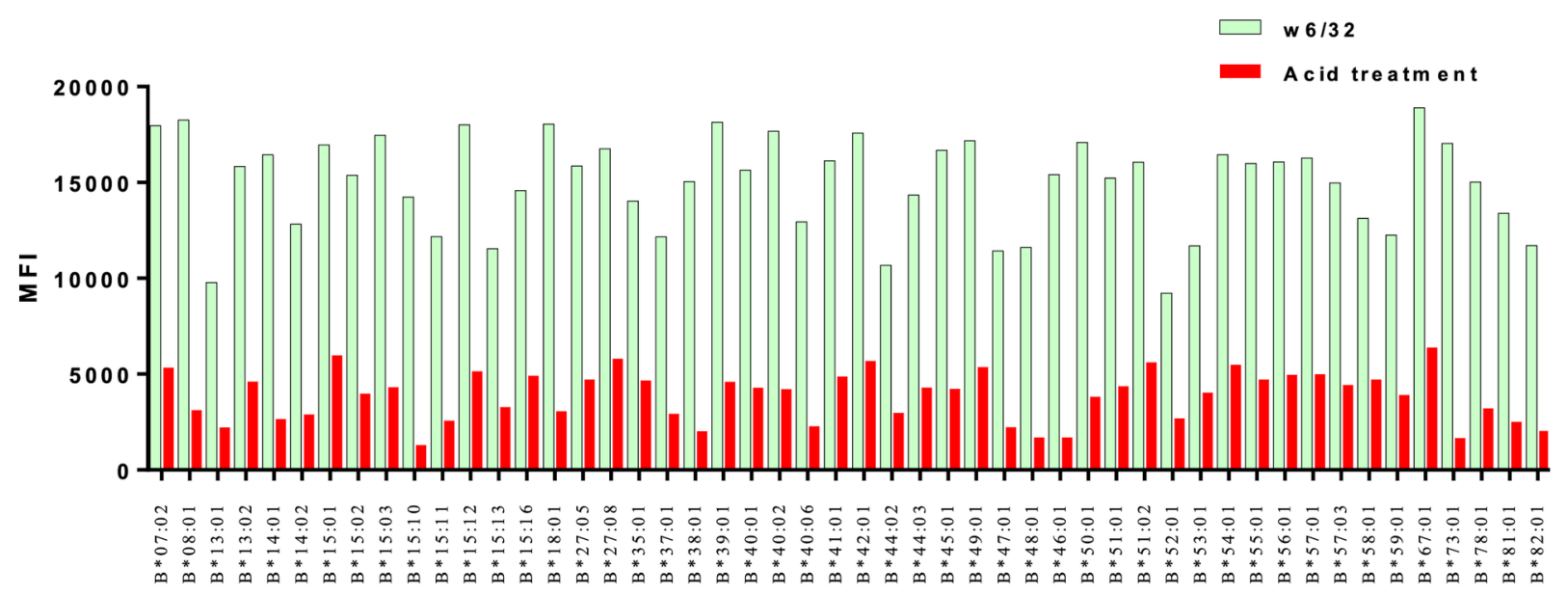




\section{Appendix Figure 2C}

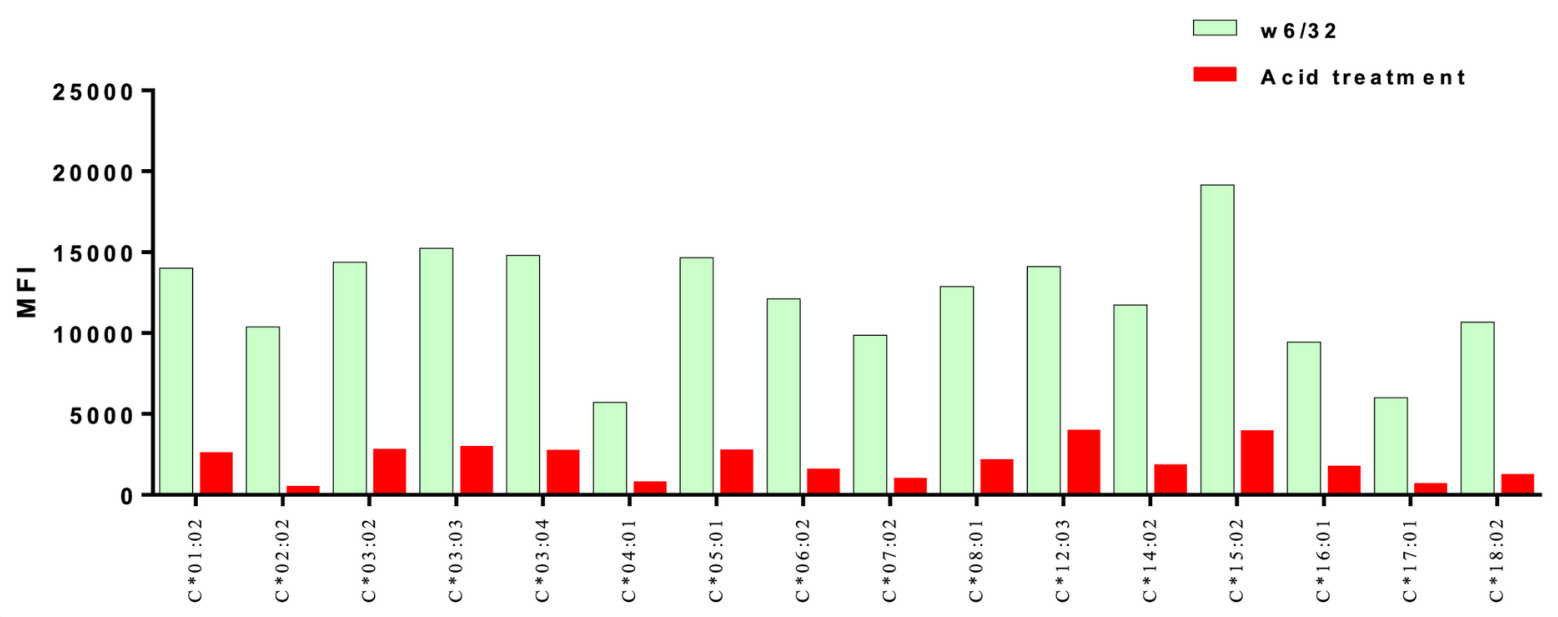

This article is protected by copyright. All rights reserved. 
endix Table1. Demographic and clinical parameters of prospective cohort.

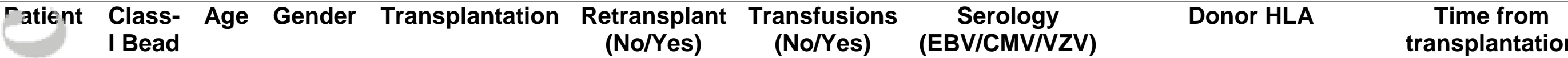 \\ (months)}

\begin{tabular}{|c|c|c|c|c|c|c|c|c|c|}
\hline 1 & B7 & 68 & Male & Kidney & No & Yes & $+/+/+$ & $\mathrm{A} 2, \mathrm{~A} 23, \mathrm{~B} 18, \mathrm{~B} 57$ & 10 \\
\hline$\angle$ & A23 & 60 & Female & Kidney & No & Yes & $+/+/+$ & $\mathrm{A} 2, \mathrm{~A} 3, \mathrm{~B} 7, \mathrm{~B} 12$ & 309 \\
\hline 2 & B17 & 52 & Female & No & No & No & $+/+/+$ & & \\
\hline 4 & A34 & 30 & Female & No & No & No & $+/+/+$ & & \\
\hline 5 & B73 & 34 & Female & No & No & No & $+/+/+$ & & \\
\hline 6 & A11 & 54 & Male & Kidney & No & Yes & $+/+/+$ & $\mathrm{A} 2, \mathrm{~A} 32, \mathrm{~B} 7, \mathrm{~B} 64$ & 5 \\
\hline 7 & Cw7 & 48 & Male & Heart & No & Yes & $+/+/+$ & A2, A26, B38,B40, & 14 \\
\hline 8 & A80 & 30 & Male & Kidney & No & No & $+/-/+$ & $\mathrm{A} 1, \mathrm{~A} 23, \mathrm{~B} 49, \mathrm{~B} 57$ & 48 \\
\hline 9 & Cw9 & 55 & Male & No & No & No & $+/+/+$ & & \\
\hline 10 & B37 & 68 & Male & No & No & Yes & $+/+/+$ & & \\
\hline 11 & A34 & 56 & Male & No & No & No & $+/-/+$ & & \\
\hline
\end{tabular}

Abbreviations: SD, Standard deviation; EBV, Epstein-Barr Virus; CMV, Cytomegalovirus; VZV, Varicella Zoster Virus. 
Appendix Table 2. Demographic and clinical parameters of comparison cohort.

Patient $\begin{gathered}\text { Class- Age Gender Transplantation } \begin{array}{c}\text { Retransplant } \\ \text { I Bead }\end{array} \\ \text { (No/Yes) }\end{gathered}$

\begin{tabular}{ccccccc}
\hline $\mathbf{1}$ & A23/24 & 56 & Female & Kidney & No & A2, A25, B18, B27 \\
\hline $\mathbf{2}$ & A23/24 & 62 & Female & Kidney & No & A11, A30, B14, B55 \\
\hline $\mathbf{3}$ & A23/24 & 68 & Female & No & No & \\
\hline $\mathbf{4}$ & A23/24 & 53 & Female & No & No & \\
\hline $\mathbf{5}$ & B57/58 & 52 & Female & Kidney & Yes & A24, A26, B44, B52 \\
\hline $\mathbf{6}$ & B57/58 & 74 & Female & No & No & \\
\hline
\end{tabular}


Appendix table 3. Description of MFI value after SAB and acid-treated bead assays in monospecific reactions.

\begin{tabular}{cccc}
\hline Serum \# & Class-I Bead & $\begin{array}{c}\text { Non-treated } \\
\text { (MFI) }\end{array}$ & $\begin{array}{c}\text { Acid treated } \\
\text { (MFI) }\end{array}$ \\
\hline $\mathbf{1}$ & B7 & 3,633 & 8,729 \\
\hline $\mathbf{2}$ & $\mathrm{A} 23$ & 3,877 & 1,541 \\
\hline $\mathbf{3}$ & $\mathrm{B} 17$ & 6,100 & 4,093 \\
\hline $\mathbf{4}$ & $\mathrm{A} 34$ & 3,861 & 2,799 \\
\hline $\mathbf{5}$ & $\mathrm{B} 73$ & 13,574 & 8,127 \\
\hline $\mathbf{6}$ & $\mathrm{A} 11$ & 5,645 & 3,769 \\
\hline $\mathbf{7}$ & $\mathrm{C}$ 7 & 3,572 & 25,296 \\
\hline $\mathbf{8}$ & $\mathrm{A} 80$ & 10,139 & 23,835 \\
\hline $\mathbf{9}$ & $\mathrm{CW} 9$ & 5,190 & 11,032 \\
\hline $\mathbf{1 0}$ & $\mathrm{B} 37$ & 2,050 & 9,464 \\
\hline $\mathbf{1 1}$ & $\mathrm{A} 34$ & 3,821 & 1,320 \\
\hline
\end{tabular}

$\mathrm{MFI}$, mean fluorescence intensity

$\mathrm{SAB}$, single antigen bead assay 


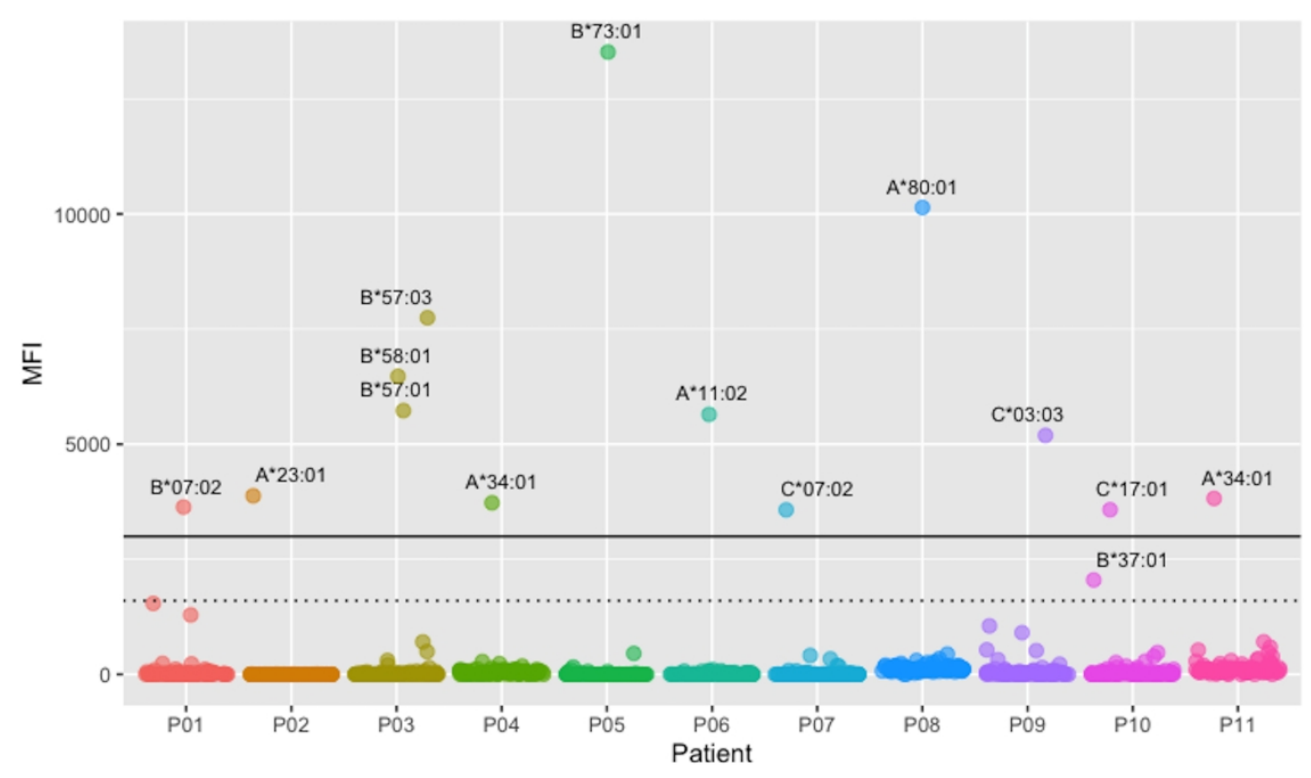

This article is protected by copyright. All rights reserved. 


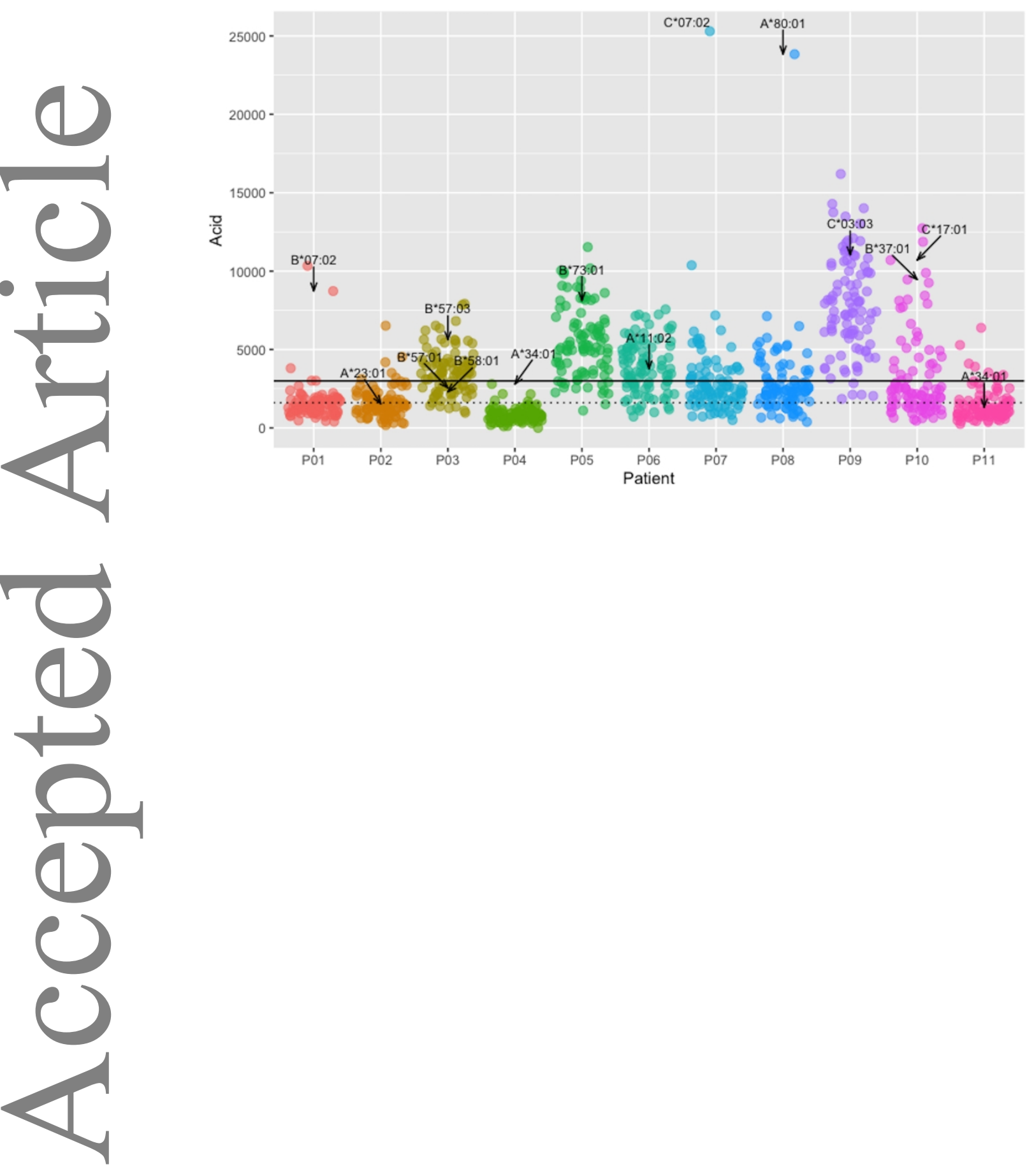

This article is protected by copyright. All rights reserved. 


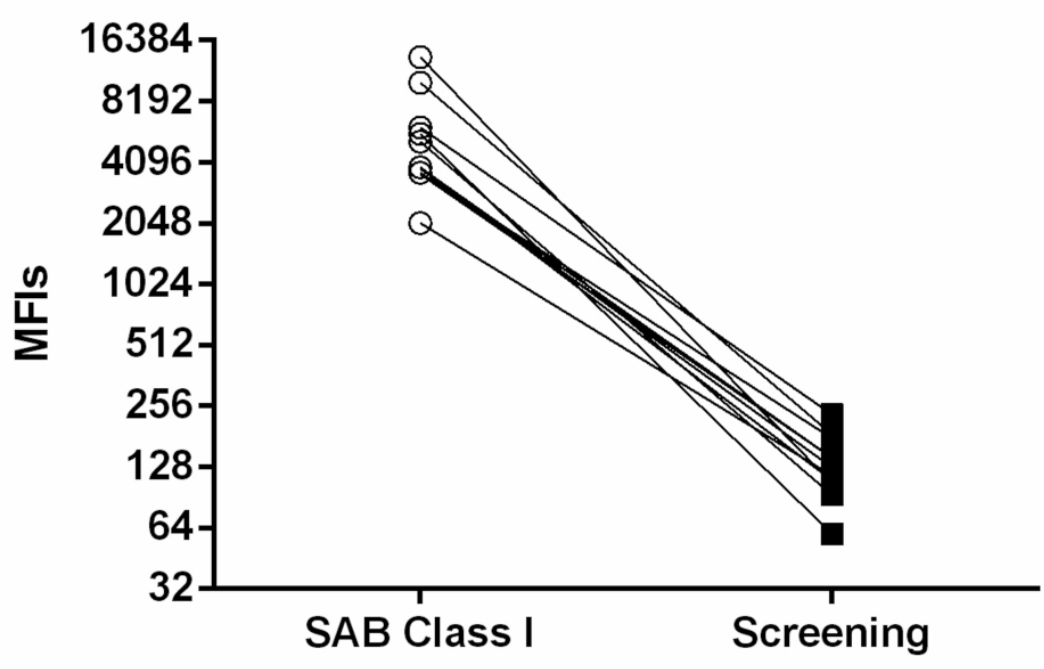

This article is protected by copyright. All rights reserved. 